Article

\title{
New Bounds for the $\alpha$-Indices of Graphs
}

\author{
Eber Lenes $^{1, *}$, Exequiel Mallea-Zepeda ${ }^{2}(\mathbb{D})$ and Jonnathan Rodríguez ${ }^{3, *} \mathbb{C}$ \\ 1 Área de Ciencias Básicas Exactas, Grupo de Investigación Deartica, Universidad del Sinú, \\ Cartagena 130001, Colombia \\ 2 Departamento de Matemática, Universidad de Tarapacá, 1000000 Arica, Chile; emallea@uta.cl \\ 3 Departamento de Matemáticas, Facultad de Ciencias Básicas, Universidad de Antofagasta, Av. Angamos 601, \\ 1240000 Antofagasta, Chile \\ * Correspondence: eber.lenes@unisinu.edu.co (E.L.); jonnathan.rodriguez@uantof.cl (J.R.)
}

Received: 1 September 2020; Accepted: 24 September 2020; Published: 28 September 2020

check for updates

\begin{abstract}
Let $G$ be a graph, for any real $0 \leq \alpha \leq 1$, Nikiforov defines the matrix $A_{\alpha}(G)$ as $A_{\alpha}(G)=$ $\alpha D(G)+(1-\alpha) A(G)$, where $A(G)$ and $D(G)$ are the adjacency matrix and diagonal matrix of degrees of the vertices of $G$. This paper presents some extremal results about the spectral radius $\rho_{\alpha}(G)$ of the matrix $A_{\alpha}(G)$. In particular, we give a lower bound on the spectral radius $\rho_{\alpha}(G)$ in terms of order and independence number. In addition, we obtain an upper bound for the spectral radius $\rho_{\alpha}(G)$ in terms of order and minimal degree. Furthermore, for $n>l>0$ and $1 \leq p \leq\left\lfloor\frac{n-l}{2}\right\rfloor$, let $G_{p} \cong K_{l} \vee\left(K_{p} \cup K_{n-p-l}\right)$ be the graph obtained from the graphs $K_{l}$ and $K_{p} \cup K_{n-p-l}$ and edges connecting each vertex of $K_{l}$ with every vertex of $K_{p} \cup K_{n-p-l}$. We prove that $\rho_{\alpha}\left(G_{p+1}\right)<\rho_{\alpha}\left(G_{p}\right)$ for $1 \leq p \leq\left\lfloor\frac{n-l}{2}\right\rfloor-1$.
\end{abstract}

Keywords: spectral radius; minimal degree; independence number; $\alpha$-adjacency matrix

\section{Introduction}

Let $G=(V(G), E(G))$ be a simple and undirected connected graph with vertex set $V(G)=\left\{v_{1}, v_{2}, \ldots, v_{n}\right\}$ and edge set $E(G)=\left\{e_{1}, e_{2}, \ldots, e_{m}\right\}$. To simplify the notation, we represent a vertex just by $u$ and then an edge is just represented by $u v$, we say that $u$ is adjacent to $v$, or that $u$ and $v$ are neighbors and we write $u \sim v$. The adjacency matrix of $G$, denoted by $A(G)=\left(a_{i j}\right)$ is a symmetric matrix of order $n$ such that $a_{i j}=1$ if $v_{i}$ and $v_{j}$ are adjacent, and 0 otherwise. Let $d_{i}=d_{v_{i}}=d_{G}\left(v_{i}\right)$ be the degree of vertex $v_{i}$ in $G$. The largest and smallest vertex degrees of $G$ are denoted by $\Delta(G)$ and $\delta(G)$, respectively. The degree matrix of $G$, denoted by $D(G)$, is the diagonal matrix with diagonal entries the vertex degrees of $G$. A $k$-regular graph is a graph where every vertex has degree $k$. The complement of a graph $G$ is represented by $\bar{G}$. The spectrum of a matrix $M$, will be denoted by $S p(M)$. In this paper, the complete graph of order $n$ is denoted by $K_{n}$. An independent set is a set of vertices in a graph, where no two vertices are adjacent. The independence number of a graph $G$ is the number of vertices of the largest independent set in $G$, denoted by $\gamma(G)$ or just $\gamma$ if there is no ambiguity. The line graph of a graph $G$, denoted by $L_{G}$, is the graph whose vertex set is the edge set of $G$, where two vertices of $L_{G}$ are adjacent, if and only if, the corresponding edges are incident in $G$. The signless Laplacian matrix and Laplacian matrix of $G$ are defined as $Q(G)=D(G)+A(G)$ and $L(G)=D(G)-A(G)$, respectively. We denote by $\mu_{1}$ the Laplacian spectral radius of $G$, by $q_{1}$ the signless Laplacian spectral radius of $G$, and by $\lambda_{1}$ the adjacency spectral radius or spectral radius of $G$. V. Nikiforov in Reference [1], define the matrix

$$
A_{\alpha}(G)=\alpha D(G)+(1-\alpha) A(G), \text { with } 0 \leq \alpha \leq 1 .
$$


Its straightforward verified that

and

$$
A_{0}(G)=A(G), 2 A_{\frac{1}{2}}(G)=Q(G), A_{1}=D(G),
$$

$$
A_{\alpha}(G)-A_{\beta}(G)=(\alpha-\beta) L(G) .
$$

We denote by $\rho_{\alpha}(G)$ to the spectral radius of $A_{\alpha}(G)$ or well called the $\alpha$-index of $G$. The join of two vertex disjoint graphs $G_{1}$ and $G_{2}$ is the graph obtained from the disjoint union $G_{1} \cup G_{2}$ by adding new edges from each vertex in $G_{1}$ to every vertex in $G_{2}$. It is usually denoted by $G_{1} \vee G_{2}$. This graph operation can be generalized in the following way: Let $H$ be a graph of order $k$ and $V(H)=\{1,2, \ldots, k\}$. Let $\mathcal{F}=\left\{G_{1}, G_{2}, \ldots, G_{k}\right\}$ be a set of pairwise vertex disjoint graphs. Here, each vertex $j \in V(H)$ is assigned to the graph $G_{j} \in \mathcal{F}$. Let $G$ be the graph obtained from the graphs $G_{1}, G_{2}, \ldots, G_{k}$ and the edges connecting each vertex of $G_{i}$ with all the vertices of $G_{j}$, for all edge $i j \in E(H)$. That is, $G$ is the graph with vertex set

$$
V(G)=\bigcup_{i=1}^{k} V\left(G_{i}\right)
$$

and edge set

$$
E(G)=\left(\bigcup_{i=1}^{k} E\left(G_{i}\right)\right) \bigcup\left(\bigcup_{i j \in E(H)}\left\{u v: u \in V\left(G_{i}\right), v \in V\left(G_{j}\right)\right\}\right) .
$$

This graph is designated the $H-$ join (or generalized composition) of the graphs $G_{1}, G_{2}, \ldots, G_{k}[2-4]$, and it is denoted by

$$
G=\bigvee_{H}\left\{G_{j}: 1 \leq j \leq k\right\}
$$

In Reference [5], the authors determine the unique graph with maximal $\alpha$-index among all connected graphs with diameter $d$, and determine the unique graph with minimal $\alpha$-index among all connected graphs with given clique number. In Reference [6], the extremal graph with maximal $\alpha$-index with fixed order and cut vertices and the extremal tree which attains the maximal $\alpha$-index with fixed order and matching number are characterized. In Reference [7], the authors obtain the extremal graphs with maximal $\alpha$-index with fixed order and diameter at least $k$. In References [8,9], are characterized the graphs which have the minimal spectral radius among all the connected graphs of order $n$ and some values of the independence number $\gamma$. In Reference [10], Nikiforov et al. shown that if $T_{\Delta}$ is a tree of maximal degree $\Delta$, then the spectral radius of $A_{\alpha}\left(T_{\Delta}\right)$ satisfies the tight inequality

$$
\rho_{\alpha}\left(T_{\Delta}\right)<\alpha \Delta+2(1-\alpha) \sqrt{\Delta-1}
$$

In Reference [11], the authors obtain the following sharp upper bound for the spectral radius of $G$.

Theorem 1 ([11]). Let $G$ be a simple graph with $n$ vertices and $m$ edges. Let $\delta=\delta(G)$ be the minimum degree of vertices of $G$ and $\lambda_{1}(G)$ be the spectral radius of the adjacency matrix of $G$. Then,

$$
\lambda_{1}(G) \leq \frac{\delta-1+\sqrt{(\delta+1)^{2}+4(2 m-\delta n)}}{2} .
$$

Equality holds, if and only if, $G$ is either a regular graph or a bidegreed graph where each vertex has degree either $\delta$ or $n-1$. 
In Reference [12], is presented a sharp lower bound on the signless Laplacian spectral radius of a graph in terms of independence number.

Theorem 2 ([12]). Let $G$ be a graph of order $n$ and $\gamma(G)=\gamma$. Then

$$
q_{1}(G) \geq \frac{2(n-\gamma)}{\gamma}
$$

with equality, if and only if, $n=k \gamma$ and $G \cong \gamma K_{k}$.

This paper is organized in the following way. In Section 1, besides the main concepts and notation used throughout the paper we present some recent work that motivated the authors. In Section 2, for $n=t \gamma+s$ where $0 \leq s<\gamma$ and $0 \leq \alpha<1$, a lower bound for the $\alpha$-index of graphs of order $n$ and independence number $\gamma$ is given, further it is shown equality cases.

In Section 3, for $0 \leq \alpha<1$, an upper bound for the $\alpha$-index of graphs of order $n$, edge number $m$ and minimal degree $\delta$ is given, we demonstrate that the equality holds, if and only if, is either $G$ a regular graph or a bidegreed graph where each vertex is of degree either $\delta$ or $n-1$. In Section 4 , for $0 \leq \alpha<1$, we present an ordering of $\alpha$-index in the class of the graphs $K_{l} \vee\left(K_{p} \cup K_{n-p-l}\right)$ where $1 \leq p \leq\left\lfloor\frac{n-l}{2}\right\rfloor$.

\section{Spectral Radius and Independence Number}

In this section, we present a lower bound for the spectral radius of graphs in terms of the order and independence number which generalizes and improves the lower bound presented in Theorem 2 . We first present some lemmas used in the proof of our result. For general properties of the matrices $A_{\alpha}(G)$ we refer the reader to Reference [1]. In particular, we frequently use the facts that $\rho_{\alpha}(G)$ is non-decreasing in $\alpha$ ([1], Proposition 4).

Lemma 1 ([1]). Let $G$ be a graph of order $n$ and $H$ be any subgraph of $G$. If $0 \leq \alpha \leq 1$ then

$$
\rho_{\alpha}(H) \leq \rho_{\alpha}(G)
$$

Remark 1 ([12]). If $S \subseteq V(G)$ is a maximal independent set of $G$, then for each $u \in V(G)-S$, there exists some vertex $v \in S$ such that $u v \in E(G)$. Moreover, for any $w \in V(G)-(S \cup\{u\})$ with $u w \notin E(G)$ and $w v \in E(G)$, there exists some vertex $v_{1} \in S-v$ such that $u v_{1} \in E(G)$ or $w v_{1} \in E(G)$.

Remark 2 ([12]). For a graph $G$ on $n$ vertices non-isomorphic to the graph $\bar{K}_{n}$, we have

$$
\gamma(G) \leq \gamma(G-e) \leq \gamma(G)+1
$$

Lemma 2 ([13]). Let $G$ be a graph of order $n$ and $m$ edges. Let $0 \leq \alpha<1$. Then,

$$
\rho_{\alpha}(G) \geq \frac{2 m}{n}
$$

with equality, if and only if, $G$ is a regular graph.

Lemma 3. Let $G$ be a graph of order $n$ and independence number $\gamma$ with $n=t \gamma+s$ where $0 \leq s<\gamma$ and $1<\gamma<n$. Then $G \cong s K_{t+1} \cup(\gamma-s) K_{t}$, if and only if, in $G$ the following conditions are verified

(a) there exists t maximal independent sets $S_{1}, \ldots, S_{t}$ and an independent set $S_{t+1}$ of cardinality s where $S_{i} \cap S_{j}=$ $\phi$ for $i, j=1, \ldots, t+1, i \neq j$ 
(b) each vertex of $S_{i}$ is adjacent to a single vertex of $S_{j}$ for $i, j=1, \ldots, t+1, i \neq j$.

Proof. Let $G$ be a graph of order $n$ and independence number $\gamma$ that verifies the conditions (a) and (b). If $t=1$ and $s>0$ or $t=2$ and $s=0$ then $G \cong s K_{t+1} \cup(\gamma-s) K_{t}$, the result holds. Otherwise, let $x \in S_{k}$, $y \in S_{l}$ and $z \in S_{m}$ where $k=1, \ldots, t+1 ; l, m=1, \ldots, t$ and $k \neq m \neq l \neq k$. Suppose $x y \in E(G)$ and $x z \in E(G)$. We claim $y z \in E(G)$. Suppose $y z \notin E(G)$ then by (b) $z w \in E(G)$ for some $w \in S_{l}, w \neq y$. By (a) $x \neq w$. Then, by (b) $\left(S_{m}-\{z\}\right) \cup\{x, w\}$ would be an independent set in $G$ of cardinality $\gamma+1$ which is a contradiction. By repeated applications of the above argument we can conclude that $G \cong s K_{t+1} \cup(\gamma-s) K_{t}$. Conversely, if $G \cong s K_{t+1} \cup(\gamma-s) K_{t}$ then $G$ verify the conditions (a) and (b). The proof is complete.

Lemma 4. Let $G \neq s K_{t+1} \cup(\gamma-s) K_{t}$ be a graph of order $n$ and independence number $\gamma$ with $n=t \gamma+s$ where $0 \leq s<\gamma$ and $1<\gamma<n$, then $G$ has a proper subgraph $H$ of order $n$ and independence number $\gamma$.

Proof. Suppose $G \nRightarrow s K_{t+1} \cup(\gamma-s) K_{t}$ then $G$ does not verify some of the conditions (a) or (b) of Lemma 3 . Suppose $G$ does not verify the condition (a), then the following two cases may occur:

(i) $G$ has exactly $\ell$ maximal independent sets $S_{1}, \ldots, S_{\ell}$ where $\ell<t$ and $S_{i} \cap S_{j}=\phi$ for $i, j=1, \ldots, \ell$, $i \neq j$. Then, $\gamma\left(G-\bigcup_{i=1}^{\ell} S_{i}\right)<\gamma$ (otherwise, if $\gamma\left(G-\bigcup_{i=1}^{\ell} S_{i}\right)=\gamma$ then $G$ would have $\ell+1$ two by two disjoint independent sets which would be a contradiction). Then, by Remark 2, we can constructed a new graph $H_{1} \cong G-e$ on $n$ vertices and independence number $\gamma$, where $e \in E\left(G-\bigcup_{i=1}^{\ell} S_{i}\right)$, the result holds.

(ii) $G$ has exactly $t$ maximal independent sets $S_{1}, \ldots, S_{t}$ and $S_{i} \cap S_{j}=\phi$ for $i, j=1, \ldots, t, i \neq j, s>0$ and $G_{1} \cong G-\bigcup_{i=1}^{t} S_{i} \neq \bar{K}_{s}$ then we can constructed a new graph $H_{2} \cong G-e$ on $n$ vertices and independence number $\gamma$, where $e \in E\left(G_{1}\right)$, the result holds.

Now, suppose that $G$ verify condition (a) but does not verify condition (b), this is, we assume that $G$ has $t$ maximal independent sets $S_{1}, \ldots, S_{t}$ and an independent set $S_{t+1}$ of cardinality s such that $S_{i} \cap S_{j}=\phi$ for $i, j=1, \ldots, t+1$ with $i \neq j$. As $G$ does not check condition (b) there exists $v \in S_{i}$ and $u, w \in S_{j}$ for some $i, j=1, \ldots, t+1, i \neq j$ and $u \neq w$ such that $u v \in E(G)$ and $w v \in E(G)$.

(i) For $i=1, \ldots, t$, by Remark 1 , there exists some vertex $v_{1} \in S_{i}-\{v\}$ such that $u v_{1} \in E(G)$ or $w v_{1} \in E(G)$. Suppose $u v_{1} \in E(G)$. Then, we constructed a new graph $H_{3} \cong G-u v$ on $n$ vertices and independence number $\gamma$, the result holds.

(ii) Suppose that each vertex of $S_{i}$ is adjacent to a single vertex of $S_{j}$ for $i=1, \ldots, t$ and $j=1, \ldots, t+1$, $i \neq j$, then we use the same techniques applied in the proof from Lemma 3, we can see that $G-S_{t+1} \cong \gamma K_{t}$. Now, let $i=t+1$ and $G-S_{t+1} \cong \gamma K_{t}$. We claim, $v$ is adjacent to all the vertices of a connected component of $G-S_{t+1}$ isomorphic to $K_{t}$. Otherwise, there exists $v_{m}$ in each connected component of $G-S_{t+1}$ isomorphic to $K_{t}$ such that $v v_{m} \notin E(G)$ for $m=1, \ldots, \gamma$. So, $S=\left\{v, v_{1}, \ldots, v_{\gamma}\right\}$ would be an independent set in $G$ of cardinality $\gamma+1$ which is a contradiction. Then, there exists $Z=\left\{v_{1}, \ldots, v_{t}\right\}$ such that $v_{p} \in V(G)-S_{t+1}, v v_{p} \in E(G)$ for $p=1, \ldots, t$ and $v_{p} v_{q} \in E(G)$ for $p, q=1, \ldots, t$. Thus, $u \notin Z$ or $w \notin Z$. If $u \notin Z$ then we constructed a new graph $H_{4} \cong G-u v$ of order $n$ and independence number $\gamma$.

The proof is complete.

Let $G$ and $H$ be two graphs of order $n$, we will say that $G$ and $H$ are comparable, if and only if, $H$ is subgraph of $G$ or $G$ is subgraph of $H$. 
Lemma 5. Let $G$ be a graph of order $n$ and independence number $\gamma$ with $n=t \gamma+s$ where $0 \leq s<\gamma$ and $1<\gamma<n$, then $s K_{t+1} \cup(\gamma-s) K_{t}$ is a subgraph of $G$.

Proof. Let $G_{1} \cong s K_{t+1} \cup(\gamma-s) K_{t}$ then by Lemma 3 in $G_{1}$ the following conditions are verified (a) there exists $t$ maximal independent sets $S_{1}, \ldots, S_{t}$ and an independent set $S_{t+1}$ of cardinality $s$ where $S_{i} \cap S_{j}=\phi$ for $i, j=1, \ldots, t+1, i \neq j$ and (b) each vertex of $S_{i}$ is adjacent to a single vertex of $S_{j}$ for $i, j=1, \ldots, t+1$, $i \neq j$. Let $G_{2} \cong G_{1}-u v$ where $u \in S_{i}, v \in S_{j}$ and $u v \in E\left(G_{1}\right)$ for some $i, j=1, \ldots, t+1, i \neq j$. Thus, in $G_{2}$ some of the sets $S_{i} \cup\{v\}$ or $S_{j} \cup\{u\}$ is an independent set of cardinality $\gamma+1$. Since $G$ is a graph of order $n$ and independence number $\gamma$ then $G$ is not a proper subgraph of $G_{1}$. Now, suppose $G$ and $G_{1}$ are not comparable graphs. By Lemma $4, G$ has a proper subgraph $H_{1}$ of order $n$ and independence number $\gamma$. Clearly, $H_{1} \nsucceq G_{1}$. Then by Lemma $4, H_{1}$ has a proper subgraph $H_{2}$ of order $n$ and independence number $\gamma$. Clearly, $H_{2} \neq G_{1}$. By repeated applications of previous argument, we can conclude $\bar{K}_{n}$ is a graph of orden $n$ and independence number $\gamma$ where $1<\gamma<n$ which is a contradiction. Hence, $G$ and $G_{1}$ are comparable graphs. Since $G$ is not a proper subgraph of $G_{1}$ then $G_{1}$ is a subgraph of $G$. The proof is complete.

Below we present the main result of the section which is a lower bound for the spectral radius of graphs in terms of order and independence number.

Theorem 3. Let $G$ be a graph of order $n$ and independence number $\gamma$ with $n=t \gamma+s$ where $0 \leq s<\gamma$. Let $0 \leq \alpha<1$ then

$$
\rho_{\alpha}(G) \geq \begin{cases}\frac{n-s}{\gamma} & \text { if } s>0, \\ \frac{n-\gamma}{\gamma} & \text { if } s=0 .\end{cases}
$$

- If $s=0$, then the equality holds, if and only if, $G \cong \gamma K_{t}$.

- If $s>0$, then the equality holds if $G \cong s K_{t+1} \cup(\gamma-s) K_{t}$.

Proof. Let $G$ be a graph of order $n$ and independence number $\gamma$ with $n=t \gamma+s$ where $0 \leq s<\gamma$. First consider $\gamma=1$ then $s=0$ and $n=t$. By Remark $1, G \cong K_{n} \cong \gamma K_{t}$ the result holds. Now, let $\gamma=n$ then $s=0$ and $t=1$. Then, $G \cong \bar{K}_{n} \cong \gamma K_{t}$ the result is true. Now, we assume $1<\gamma<n$. By Lemma 5, $s K_{t+1} \cup(\gamma-s) K_{t}$ is a subgraph of $G$. Clearly, for $G^{*} \cong s K_{t+1} \cup(\gamma-s) K_{t}$, we have $\rho_{\alpha}\left(G^{*}\right)=\frac{n-s}{\gamma}$ if $s>0$, and $\rho_{\alpha}\left(G^{*}\right)=\frac{n-\gamma}{\gamma}$ if $s=0$. By Lemma 1 ,

$$
\rho_{\alpha}(G) \geq \begin{cases}\frac{n-s}{\gamma} & \text { if } s>0 \\ \frac{n-\gamma}{\gamma} & \text { if } s=0\end{cases}
$$

Suppose the equality holds, $s=0$ and $G \neq G^{*}$ then the edge number $m$ of $G$ is greater than $\frac{\gamma t(t-1)}{2}$. By Lemma 2, $\rho_{\alpha}(G) \geq \frac{2 m}{n}>\frac{\gamma t(t-1)}{n}=\frac{n-\gamma}{\gamma}$ which is a contradiction. The proof is complete.

The following result which is a direct consequence of Theorem 3, presents a lower bound for the signless Laplacian spectral radius and this in turn improves the bound of Theorem 2. 
Corollary 1. Let $G$ be a graph of order $n$ and independence number $\gamma$ with $n=t \gamma+s$ where $0 \leq s<\gamma$, then

$$
q_{1}(G) \geq \begin{cases}\frac{2(n-s)}{\gamma} & \text { if } s>0, \\ \frac{2(n-\gamma)}{\gamma} & \text { if } s=0 .\end{cases}
$$

- If $s=0$, then the equality holds, if and only if, $G \cong \gamma K_{t}$.

- If $s>0$, then the equality holds if $G \cong s K_{t+1} \cup(\gamma-s) K_{t}$.

Proof. Taking $\alpha=\frac{1}{2}$ then $2 A_{\alpha}(G)=Q(G)$, by Theorem 3 the result holds.

\section{Spectral Radius and Minimal Degree}

In this section, we provide the necessary definitions and lemmas on which our main results rely. We begin with some simple matrix results. Let $B=\left(b_{i j}\right)$ be an $m \times n$ matrix. Then $s_{i}(B)$ will denote the $i$-th row sum of $B$, that is, $s_{i}(B)=\sum_{j=1}^{n} b_{i j}$, where $1 \leq i \leq m$.

Lemma 6 ([14]). Let $B$ be a real symmetric $n \times n$ matrix and $\lambda$ be an eigenvalue of $B$, with an eigenvector $x$ all of whose entries are nonnegative. Then

$$
\min _{1 \leq i \leq n} s_{i}(B) \leq \lambda \leq \max _{1 \leq i \leq n} s_{i}(B)
$$

Moreover, if the row sums of $B$ are not all equal and if all entries of $x$ are positive, then both inequalities above are strict.

An immediate consequence is the following result.

Lemma 7. Let $G$ be a simple connected graph of order $n$ with $A_{\alpha}(G)=A_{\alpha}$ where $0 \leq \alpha<1$. Let $P$ be any polynomial and $R_{v}\left(P\left(A_{\alpha}\right)\right)$ be the row sum of $P\left(A_{\alpha}\right)$ corresponding to $v \in V(G)$, then

$$
\min _{v \in V(G)} R_{v}\left(P\left(A_{\alpha}\right)\right) \leq P\left(\rho_{\alpha}(G)\right) \leq \max _{v \in V(G)} R_{v}\left(P\left(A_{\alpha}\right)\right) .
$$

Both equalities above holds, if and only if, the row sums of $P\left(A_{\alpha}\right)$ are all equal.

As a consequence of Lemma 7, we obtain the following result which generalize Theorem 1.

Theorem 4. Let $G$ be a simple connected graph of order $n$ and minimal degree $\delta$ with m edges and $0 \leq \alpha<1$. Then

$$
\rho_{\alpha}(G) \leq \frac{\delta-1+\alpha+\sqrt{(\delta+1-\alpha)^{2}+4(2 m-n \delta)(1-\alpha)}}{2} .
$$

The equality holds, if and only if, $G$ is either a regular graph or a bidegreed graph where each vertex has degree either $\delta$ or $n-1$. 
Proof. Let $G$ be a graph of order $n$, minimal degree $\delta$ and $m$ edges and $R_{v}\left(A_{\alpha}\right)$ be the row sum of $A_{\alpha}$ corresponding to $v \in V(G)$ where $A_{\alpha}$ denote the matrix $A_{\alpha}(G)$. We can easily see that $R_{v}\left(A_{\alpha}\right)=d_{v}$ for $v \in V(G)$. Calculating the row sum of $A_{\alpha}^{2}$ for $v \in V(G)$, we have

$$
R_{v}\left(A_{\alpha}^{2}\right)=\alpha d_{v}^{2}+(1-\alpha) \sum_{u \sim v} d_{u}=\alpha d_{v}^{2}+(1-\alpha)\left(2 m-d_{v}-\sum_{u \chi v, u \neq v} d_{u}\right)
$$

Thus,

$$
R_{v}\left(A_{\alpha}^{2}\right) \leq \alpha d_{v}^{2}+(1-\alpha)\left(2 m-d_{v}-\left(n-d_{v}-1\right) \delta\right)
$$

Thereby,

$$
R_{v}\left(A_{\alpha}^{2}\right)-\left((1-\alpha)(\delta-1)+\alpha d_{v}\right) R_{v}\left(A_{\alpha}\right) \leq(1-\alpha)(2 m-(n-1) \delta) .
$$

For the linearity,

$$
R_{v}\left(A_{\alpha}^{2}-\left((1-\alpha)(\delta-1)+\alpha d_{v}\right) A_{\alpha}\right) \leq(1-\alpha)(2 m-(n-1) \delta) .
$$

Taking $P(x)=x^{2}-\left((1-\alpha)(\delta-1)+\alpha d_{v}\right) x$ in Lemma 7, we have

$$
\rho_{\alpha}^{2}(G)-\left((1-\alpha)(\delta-1)+\alpha d_{v}\right) \rho_{\alpha}(G)-(1-\alpha)(2 m-(n-1) \delta) \leq 0 .
$$

In particular,

$$
\rho_{\alpha}^{2}(G)-(\delta-1+\alpha) \rho_{\alpha}(G)-(1-\alpha)(2 m-(n-1) \delta) \leq 0 .
$$

Solving the inequality (2) for $\rho_{\alpha}(G)$, we obtain

$$
\rho_{\alpha}(G) \leq \frac{\delta-1+\alpha+\sqrt{(\delta+1-\alpha)^{2}+4(2 m-n \delta)(1-\alpha)}}{2} .
$$

Suppose the equality in (3) holds, then all the inequalities in the above argument must be equalities. This implies that

$$
2 m-d_{v}-\left(n-d_{v}-1\right) \delta=\sum_{u \sim v} d_{u}
$$

for all $v \in V(G)$, that is,

$$
\sum_{u \sim v, u \neq v} d_{u}=\left(n-d_{v}-1\right) \delta
$$

for all $v \in V(G)$. Now, suppose $u_{1}$ and $v_{1}$ are two non-adjacent vertices in $G$ then $d_{v_{1}}<n-1$. By (4), we have $d_{u_{1}}=\delta$. Analogously, we can prove that $d_{v_{1}}=\delta$. Hence, the affirmation "Any pair of non-adjacent vertices in $G$ have degree $\delta^{\prime \prime}$ is true. Now, if there exists $v \in V(G)$ such that $d_{v}>\delta$ then by above affirmation, we conclude $v$ is adjacent to $u$ for all $u \in V(G)$ with $u \neq v$, this is, $d_{v}=n-1$ which implies either $G$ is a regular graph of degree $\delta$ or $G$ is a bidegreed graph where each vertex has degree either $\delta$ or $n-1$. Conversely, if $G$ is a regular graph then the equality holds. Now, suppose $G$ is a bidegreed graph where each vertex has degree $\delta$ or $n-1$. 
- $\quad$ Let $v \in V(G)$ such that $d_{v}=n-1$ then $\sum_{u \neq v, u \neq v} d_{u}=0$, since $R_{v}\left(A_{\alpha}\right)=d_{v}$, by (1) we have

$$
\begin{aligned}
R_{v}\left(A_{\alpha}^{2}\right)-\left((1-\alpha)(\delta-1)+\alpha d_{v}\right) R_{v}\left(A_{\alpha}\right) & =(1-\alpha)\left(2 m-d_{v}-(\delta-1) d_{v}\right) \\
& =(1-\alpha)(2 m-(n-1) \delta) .
\end{aligned}
$$

- $\quad$ Let $v \in V(G)$ such that $d_{v}=\delta$, since $R_{v}\left(A_{\alpha}\right)=d_{v}$, by (1) and (4) we have

$$
\begin{aligned}
R_{v}\left(A_{\alpha}^{2}\right)-\left((1-\alpha)(\delta-1)+\alpha d_{v}\right) R_{v}\left(A_{\alpha}\right) & =(1-\alpha)\left(2 m-d_{v}-\sum_{u \neq v, u \neq v} d_{u}-(\delta-1) d_{v}\right) \\
& =(1-\alpha)(2 m-\delta-(n-1-\delta) \delta)-(\delta-1) \delta) \\
& =(1-\alpha)(2 m-(n-1) \delta) .
\end{aligned}
$$

Now, taking $P(x)=x^{2}-\left((1-\alpha)(\delta-1)+\alpha d_{v}\right) x$ in Lemma 7 , we have

$$
\rho_{\alpha}^{2}(G)-\left((1-\alpha)(\delta-1)+\alpha d_{v}\right) \rho_{\alpha}(G)-(1-\alpha)(2 m-(n-1) \delta)=0 .
$$

Hence,

$$
\rho_{\alpha}(G)=\frac{\delta-1+\alpha+\sqrt{(\delta+1-\alpha)^{2}+4(2 m-n \delta)(1-\alpha)}}{2} .
$$

The proof is complete.

Corollary 2. Let $G$ be a graph of order $n$ and minimal degree $\delta$ with $m$ edges then

$$
q_{1} \leq \frac{2 \delta-1+\sqrt{8(2 m-n \delta)+(2 \delta+1)^{2}}}{2} .
$$

Equality holds, if and only if, $G$ is either a regular graph or a bidegreed graph where each vertex has degree either $\delta$ or $n-1$.

Proof. Taking $\alpha=\frac{1}{2}$ then $2 A_{\alpha}(G)=Q(G)$, by Theorem 4 the result holds.

Lemma $8([15,16])$. Let $G$ be a graph of order $n$ with $m \geq 1$ edges. Let $q_{i}$ be the $i$-th greatest signless Laplacian eigenvalue of $G$ and $\lambda_{i}\left(L_{G}\right)$ be the $i$-th greatest eigenvalue of the line graph of $G$. Then

$$
q_{i}=\lambda_{i}\left(L_{G}\right)+2,
$$

for $i=1,2, \ldots, k$, where $k=\min \{n, m\}$. In addition, if $m>n$, then $\lambda_{i}\left(L_{G}\right)=-2$ for $i \geq n+1$ and if $n>m$, then $q_{i}=0$ for $i \geq m+1$.

As a direct consequence of Corollary 2 and Lemma 8, we obtain the following result.

Corollary 3. Let $G$ be a graph of order $n$ and minimal degree $\delta$ with $m$ edges then

$$
\lambda_{1}\left(L_{G}\right) \leq \frac{2 \delta-5+\sqrt{8(2 m-n \delta)+(2 \delta+1)^{2}}}{2} .
$$


Equality holds, if and only if, $G$ is either a regular graph or a bidegreed graph where each vertex has degree $\delta$ or $n-1$.

\section{Monotonicity of the $\alpha$-Indices of Graphs with $0 \leq \alpha<1$}

In this section, for $0 \leq \alpha<1$, we present an ordering of $\alpha$-indices in the class of the graphs $G_{p} \cong K_{l} \vee\left(K_{p} \cup K_{n-p-l}\right)$ where $1 \leq p \leq\left\lfloor\frac{n-l}{2}\right\rfloor$. The graphs $G_{p}$ plays an important role in the representation of graphs that relate the join operation between an arbitrary family of graphs, in particular maximize some topological indices of graphs in terms of edge connectivity and vertices connectivity such as Energy, Estrada index, Spread (see References [17-19]). Furthermore, in graphs of communication or transportation networks, the edge connectivity is an important measure of reliability, the study of the line graph of these graphs is relevant. In Theorem 8 [20], for $1 \leq p \leq\left\lfloor\frac{n-l}{2}\right\rfloor$ is presented an ordering between the spectral radii of the distance matrices $\mathcal{D}_{\alpha}\left(G_{p}\right)$ where $\alpha \in(\bar{\alpha}, 1)$, being $\bar{\alpha}=\max \left\{\alpha_{p}: 2 \leq p \leq\left\lfloor\frac{n-l}{2}\right\rfloor\right\}$ and $\alpha_{p}$ the unique zero of the function $e(x, \alpha)=(4 \alpha-3) x+\left(\alpha^{2}(2 l-4 n)+\alpha(3 n-3 l+4)+l-3\right)$ in the interval $\left(\frac{3}{4}, \frac{3 n-l}{4 n-2 l}\right)$. In this section, we prove that this same order is conserved for the spectral radii of the matrices $A_{\alpha}\left(G_{p}\right)$ with $\alpha \in[0,1)$. We begin presenting a result for the spectrum of the matrices $A_{\alpha}\left(G_{p}\right)$ with $\alpha \in[0,1)$. In Theorem 5 [2], the spectrum of the adjacency matrix of the $H$-join of regular graphs is obtained. The version of this result for the matrices $A_{\alpha}$ with $0 \leq \alpha<1$ is given below, its proof is similar.

Theorem 5. Let $G \cong \bigvee_{H}\left\{G_{j}: j \in V(H)\right\}$ where $H$ is a graph of order $k$ and $G_{j}$ is a $r_{j}$-regular graph of order $n_{j}$ for $j=1, \ldots, k$. Then, the spectrum of the matrix $A_{\alpha}(G)$ with $0 \leq \alpha<1$ is

$$
\bigcup_{G_{j} \nsucceq K_{1}}\left\{\lambda: \lambda \in S p\left(A_{\alpha}\left(G_{j}\right)+\alpha s_{j} I_{n_{j}}\right)-\left\{r_{j}+\alpha s_{j}\right\}\right\} \cup S p(M(G))
$$

where $M(G)$ is the matrix of order $k$ given by

$$
M(G)=\left[\begin{array}{cccc}
\alpha s_{1}+r_{1} & \delta_{12} \sqrt{n_{1} n_{2}} & \cdots & \delta_{1 k} \sqrt{n_{1} n_{k}} \\
\delta_{12} \sqrt{n_{1} n_{2}} & \alpha s_{2}+r_{2} & \ddots & \vdots \\
\vdots & \ddots & \ddots & \delta_{(k-1) k} \sqrt{n_{k-1} n_{k}} \\
\delta_{1 k} \sqrt{n_{1} n_{k}} & \cdots & \delta_{(k-1) k} \sqrt{n_{k-1} n_{k}} & \alpha s_{k}+r_{k}
\end{array}\right]
$$

with

$$
\delta_{i j}=\left\{\begin{array}{cc}
1-\alpha & \text { if } i j \in E(H) \\
0 & \text { otherwise }
\end{array}\right.
$$

and

$$
s_{i}=\sum_{j i \in E(H)} n_{j}
$$

Let $l$ and $n$ be fixed positive integer and $p$ be a positive integer such that $1 \leq p \leq\left\lfloor\frac{n-l}{2}\right\rfloor$, by (5) we have

$$
M\left(G_{p}\right)=\left[\begin{array}{ccc}
\alpha(n-l)+l-1 & (1-\alpha) \sqrt{l p} & (1-\alpha) \sqrt{l(n-l-p)} \\
(1-\alpha) \sqrt{l p} & \alpha l+p-1 & 0 \\
(1-\alpha) \sqrt{l(n-l-p)} & 0 & n-p-(1-\alpha) l-1
\end{array}\right] .
$$

We remember that the eigenvalues of $A_{\alpha}\left(K_{s}\right)$ are $s-1$ and $\alpha s-1$ with multiplicity $s-1$. The following result is a direct consequence of Theorem 5 . 
Corollary 4. The spectrum of the matrix $A_{\alpha}\left(G_{p}\right)$ with $0 \leq \alpha<1$ and $1 \leq p \leq\left\lfloor\frac{n-l}{2}\right\rfloor$ has the eigenvalues

$$
\begin{array}{ll}
\alpha n-1 & l-1 \text { times, } \\
\alpha(l+p)-1 & p-1 \text { times, } \\
\alpha(n-p)-1 & n-l-p-1 \text { times, }
\end{array}
$$

and the eigenvalues of the matrix $M\left(G_{p}\right)$.

Below we present Rayleigh's principle for real symmetric matrices.

Lemma 9 ([21]). Let $\beta_{1}$ and $\beta_{n}$ be the largest and smallest eigenvalues of a real symmetric matrix $H$, respectively, then

$$
\beta_{1}(G)=\max \left\{x^{t} H x: x^{t} x=1\right\} \text { and } \beta_{n}(G)=\min \left\{x^{t} H x: x^{t} x=1\right\} .
$$

Moreover, if $x$ is a unit n-vector then $\beta_{1}(G)=x^{t} H x$, if and only if, $x$ is an associated eigenvector to $\beta_{1}(G)$; and $\beta_{n}(G)=x^{t} H x$, if and only if, $x$ is an associated eigenvector to $\beta_{n}(G)$.

The following remark is an immediate consequence of Lemma 9.

Remark 3. For $0 \leq \alpha<1$ and $1 \leq p \leq\left\lfloor\frac{n-l}{2}\right\rfloor$, the spectral radius of the matrix $M\left(G_{p}\right)$ is not less than its diagonal entries.

As a direct consequence of Corollary 4 and Remark 3, we have

Lemma 10. For $0 \leq \alpha<1$ and $1 \leq p \leq\left\lfloor\frac{n-l}{2}\right\rfloor$, the spectral radius of the matrix $M\left(G_{p}\right)$ is the $\alpha$-index of $G_{p}$.

One can easily verify the following result.

Lemma 11. The characteristic polynomial of $M\left(G_{p}\right)$ with $0 \leq \alpha<1$ and $1 \leq p \leq\left\lfloor\frac{n-l}{2}\right\rfloor$, is given by

$$
\begin{aligned}
f_{p, \alpha}(x) & =x^{3}+(3-n-\alpha(l+n)) x^{2}+\left(\alpha^{2} n l+\alpha((n-2)(l+n))+n(p-2)\right. \\
& -p(l+p)+3) x+l \alpha^{2}(2 p(n-l-p)-n(n-1)) \\
& +\alpha((n+l)(n-1)-p(n+3 l)(n-p-l))+p(l+1)(n-p-l)-n+1 .
\end{aligned}
$$

Theorem 6. Let $0 \leq \alpha<1$ and $1 \leq p \leq\left\lfloor\frac{n-l}{2}\right\rfloor-1$. Then,

$$
\rho_{\alpha}\left(G_{p+1}\right)<\rho_{\alpha}\left(G_{p}\right) .
$$

Proof. Since $M\left(G_{p}\right)$ and $M\left(G_{p+1}\right)$ are nonnegative irreducible matrices then its spectral radii are simple eigenvalues, we can assume $\rho_{\alpha}\left(G_{p}\right)=\eta_{1}>\eta_{2} \geq \eta_{3}$ and $\rho_{\alpha}\left(G_{p+1}\right)=\gamma_{1}>\gamma_{2} \geq \gamma_{3}$ are the eigenvalues of the matrices $M\left(G_{p}\right)$ and $M\left(G_{p+1}\right)$, respectively. 
Hence,

$$
\begin{aligned}
f_{p+1, \alpha}(x)-f_{p, \alpha}(x) & =\prod_{j=1}^{3}\left(x-\gamma_{j}\right)-\prod_{j=1}^{3}\left(x-\eta_{j}\right) \\
& =(n-l-2 p-1)\left(x+2 l \alpha^{2}-\alpha(n+3 l)+l+1\right) .
\end{aligned}
$$

We claim $\rho_{\alpha}\left(G_{p}\right)>\rho_{\alpha}\left(G_{p+1}\right)$. Otherwise, if $\rho_{\alpha}\left(G_{p}\right) \leq \rho_{\alpha}\left(G_{p+1}\right)$ then $\rho_{\alpha}\left(G_{p+1}\right) \geq \eta_{j}$, for all $j$. Taking $x=\rho_{\alpha}\left(G_{p+1}\right)$ in $(6)$, we obtain

$$
\begin{aligned}
0 & \geq-\prod_{j=1}^{3}\left(\rho_{\alpha}\left(G_{p+1}\right)-\eta_{j}\right) \\
& =(n-l-2 p-1)\left(\rho_{\alpha}\left(G_{p+1}\right)+2 l \alpha^{2}-\alpha(n+3 l)+l+1\right) \\
& \geq(n-l-2 p-1)\left(\alpha(n-l)+l-1+2 l \alpha^{2}-\alpha(n+3 l)+l+1\right) \\
& \geq 2 l(n-l-2 p-1)(\alpha-1)^{2} \\
& >0
\end{aligned}
$$

which is a contradiction. Then, we conclude $\rho_{\alpha}\left(G_{p}\right)>\rho_{\alpha}\left(G_{p+1}\right)$ for all $1 \leq p \leq\left\lfloor\frac{n-l}{2}\right\rfloor-1$.

Author Contributions: Conceptualization, E.L. and J.R.; methodology, E.L.; software, J.R.; validation, E.L., E.M.-Z. and J.R.; formal analysis, E.L.; investigation, E.M.-Z.; resources, J.R.; data curation, E.M.-Z.; writing-original draft preparation, E.L.; writing—review and editing, E.L., J.R.; visualization, J.R.; supervision, E.M.-Z.; project administration, E.M.-Z.; funding acquisition, J.R. All authors have read and agreed to the published version of the manuscript.

Funding: Eber Lenes was supported by Proyecto BASED-PD/2020-02, Universidad del Sinú, Cartagena, Colombia. Jonnathan Rodríguez was supported by MINEDUC-UA project, code ANT-1899 and Funded by the Initiation Program in Research - Universidad de Antofagasta, INI-1906. Exequiel Mallea-Zepeda was supported by Proyecto UTA-Mayor 4751-20, Universidad de Tarapacá, Arica, Chile.

Acknowledgments: The authors would like to thank the referee for his/her constructive suggestions that improved the final version of this paper

Conflicts of Interest: The authors declare no conflict of interest.

\section{References}

1. Nikiforov, V. Merging the A- and Q-spectral theories. Appl. Anal. Discret. Math. 2017, 11, 81-107. [CrossRef]

2. Cardoso, D.M.; de Freitas, M.A.; Martins, E.A.; Robbiano, M. Spectra of graphs obtained by a generalization of the join graph operation. Discret. Math. 2013, 313, 733-741. [CrossRef]

3. Cardoso, D.M.; Martins, E.; Robbiano, M.; Rojo, O. Eigenvalues of a $H$-generalized operation constrained by vertex subsets. Linear Algebra Appl. 2013, 438, 3278-3290. [CrossRef]

4. Schwenk, A.J. Computing the Characteristic Polynomial of a Graph, Graphs and Combinatorics; Lecture Notes in Mathematics; Bary, R., Harary, F., Eds.; Springer-Verlag: Berlin, Germany, 1974; Volume 406, pp. $153-172$.

5. Xue, J.; Lin, H.; Liu, S.; Shu, J. On the $A_{\alpha}$-spectral radius of a graph. Linear Algebra Appl. 2018, 550, $105-120$. [CrossRef]

6. Lin, H.; Huang, X.; Xue, J. A note on the $A_{\alpha}$-spectral radius of graphs. Linear Algebra Appl. 2018, 557, 430-437. [CrossRef]

7. Nikiforov, V.; Rojo, O. On the $\alpha$-index of graphs with pendent paths. Linear Algebra Appl. 2018, 550, 87-104. [CrossRef]

8. Li, R.; Shi, J. The minimum signless Laplacian spectral radius of graphs with given independence number. Linear Algebra Appl. 2010, 433, 1614-1622. [CrossRef] 
9. Xu, M.; Hong, Y.; Shu, J.; Zhai, M. The minimum spectral radius of graphs with a given independence number. Linear Algebra Appl. 2009, 431, 937-945. [CrossRef]

10. Nikiforov, V.; Pastén, G.; Rojo, O.; Soto, R. On the $A_{\alpha}$-spectra of trees. Linear Algebra Appl. 2017, 520, $286-305$. [CrossRef]

11. Hong, Y.; Shu, J.L.; Fang, K. A sharp upper bound of the spectral radius of graphs. J. Comb. Theory Ser. B. 2001, 81, 177-183. [CrossRef]

12. Liu, H.; Lu, M. Bounds on the independence number and signless Laplacian index of graphs. Linear Algebra Appl. 2018, 539, 44-59. [CrossRef]

13. Pirzada, S.; Rather, B.A.; Ganie, H.A. Rezwan Ul Shaban, On $\alpha$-adjacency energy of graphs and Zagreb index. arXiv 2020, arXiv:2005.01037v1.

14. Ellingham, M.N.; Zha, X. The spectral radius of graphs on surface, J. Combin. Theory Ser. B 2000, 78, 45-56. [CrossRef]

15. Brouwer, A.E.; Haemers, W.H. Spectra of Graphs; Springer-Verlag: Berlin, Germany, 2012.

16. Gutman, I.; Robbiano, M.; Cardoso, D.M.; Martins, E.A.; Medina, L.; Rojo, O. Energy of line graphs. Linear Algebra Appl. 2010, 433, 1312-1323. [CrossRef]

17. Andrade, E.; Lenes, E.; Mallea-Zepeda, E.; Robbiano, M.; Rodríguez, J. Bounds for different spreads of line and total graphs. Linear Algebra Appl. 2019, 579, 365-381. [CrossRef]

18. Andrade, E.; Lenes, E.; Mallea-Zepeda, E.; Robbiano, M.; Rodríguez, J. Extremal graphs for Estrada indices, Linear Algebra Appl. 2020, 588, 54-73. [CrossRef]

19. Lenes, E.; Mallea-Zepeda, E.; Robbiano, M.; Rodríguez, Z.J. On line graphs with maximum energy. Linear Algebra Appl. 2018, 545, 15-31. [CrossRef]

20. Díaz, R.; Pastén, G. Rojo, O. On the minimal $D_{\alpha}$-spectral radius of graphs subject to fixed connectivity. Linear Algebra Appl. 2020, 584, 353-370. [CrossRef]

21. Horn, R.; Johnson, C. Matrix Analysis; Cambridge University Press: Cambridge, UK, 1985.

(C) 2020 by the authors. Licensee MDPI, Basel, Switzerland. This article is an open access article distributed under the terms and conditions of the Creative Commons Attribution (CC BY) license (http:/ / creativecommons.org/licenses/by/4.0/). 